\title{
Comparative analysis of 3D time-resolved contrast-enhanced magnetic resonance angiography, color Doppler ultrasound and digital subtraction angiography in symptomatic carotid stenosis
}

\author{
HONGKAI CUI $^{1}$, RUIFANG YAN ${ }^{2}$, ZHANSHENG ZHAI $^{2}$, JIPENG REN ${ }^{2}$, \\ ZHENG $\mathrm{LI}^{2}$, QIANG $\mathrm{LI}^{2}$ and SHOUYING WANG ${ }^{3}$ \\ ${ }^{1}$ Department of Interventional Radiology; ${ }^{2}$ Center of Imaging, The First Affiliated Hospital \\ of Xinxiang Medical University, Weihui, Xinxiang, Henan 453100; ${ }^{3}$ School of Public Health, \\ Xinxiang Medical University, Xinxiang, Henan 453000, P.R. China
}

Received February 27, 2016; Accepted February 24, 2017

DOI: $10.3892 / \mathrm{etm} .2017 .5548$

\begin{abstract}
The present study aimed to compare the diagnostic value of contrast-enhanced magnetic resonance angiography (CE MRA) with 3D time-resolved imaging of contrast kinetics, color Doppler ultrasound (CDUS) and digital subtraction angiography (DSA) in extracranial carotid stenosis (CS). A total of 54 patients with symptomatic CS were subjected to CDUS, CE MRA and DSA examination. Results of DSA were defined as the standard, and a total of 216 vessels were examined. In each patient four vessels were examined, namely the bilateral common carotid arteries and the bilateral internal carotid arteries. The sensitivities and specificities of CE MRA and CDUS for various degrees of CS were also determined. It was observed that the sensitivities to mild-level (1-49\%), moderate-level (50-69\%) and severe-level (70-99\%) CS were $85.45,100$ and $100 \%$ for CE MRA, and 78.18, 50 and $100 \%$ for CDUS, respectively. The corresponding specificities were 95.27, 98.58 and $99.53 \%$ for CE MRA, and 79.05, 93.36 and $98.10 \%$ for CDUS, respectively. In addition, the carotid sinus plaque detection rate for CDUS was significantly higher than that of DSA and CE MRA (both $\mathrm{P}<0.001$ ). Detection rates for common carotid artery plaques and internal carotid artery plaques did not significantly differ among the three examination methods (all $\mathrm{P}>0.05$ ). These data demonstrate that $\mathrm{CE}$
\end{abstract}

Correspondence to: Professor Ruifang Yan, Center of Imaging, The First Affiliated Hospital of Xinxiang Medical University, 88 Jian Kang Road, Weihui, Xinxiang, Henan 453100, P.R. China E-mail: ruifangyandc@126.com

Professor Shouying Wang, School of Public Health, Xinxiang Medical University, 601 Jinsui Road, Xinxiang, Henan 453000, P.R. China

E-mail: shouyingwang@sina.cn

Key words: magnetic resonance angiography, contrast enhanced, color Doppler, ultrasound, digital subtraction angiography, carotid stenosis
MRA has higher sensitivity and specificity than CDUS for the diagnosis of CS, and that CDUS has a higher carotid sinus plaque detection rate than DSA and CE MRA. Therefore, the combination of MRI and CDUS may be a 'gold standard' diagnostic method for the detection of moderate and severe CS.

\section{Introduction}

Carotid stenosis (CS) is a primary cause and risk factor of ischemic cerebrovascular disease. The most common location of CS is the carotid artery bifurcation, followed by the common carotid artery, the internal carotid artery siphon, middle cerebral artery and brain arteries (1). It is generally believed that carotid artery plaques are caused by one of two mechanisms: One is severe stenosis of the carotid artery causing hemodynamic changes and correspondingly low perfusion in parts of the brain. The other is that plaque in microemboli or patches on the surface of microthrombi fall off and cause cerebral embolism (2). Previous studies indicate that $>60 \%$ of strokes are caused by carotid stenosis, and if serious these may lead to disability and even death in patients with cerebral apoplexy (3). The Society for Vascular Surgery recommends that, in patients with symptomatic CS, if noninvasive imaging identifies a stenosis degree of $>70 \%$ (class I/level A), or if angiography identifies a stenosis degree of $>50 \%$ (class I/level B), a carotid endarterectomy should be performed to reduce the risk of stroke (4). Thus, accurate assessment of the degree of CS is critical for the prevention of stroke (5). Digital subtraction angiography (DSA) is generally considered to be the gold standard for the diagnosis of CS. However, the rate of stroke induced by the invasive DSA procedure ranges from 0.5 to $1 \%(6-8)$. With the development of noninvasive magnetic resonance techniques and imaging technology, the diagnostic values of magnetic resonance angiography (MRA) and Doppler ultrasound (DUS) in CS are a current focus of research. In the diagnosis of CS, the results of these noninvasive examination methods are consistent with those of DSA; therefore, MRA and DUS, as noninvasive screening methods, may be suitable replacements for DSA (9-12). However, the reliability and accuracy of contrast-enhanced MRA (CE MRA) and color 
DUS (CDUS) in detecting the extent of CS remain to be determined by large scale studies.

In the current prospective study, CE MRA and CDUS techniques were used to examine the neck vessels and branches in symptomatic CS patients. In turn, the sensitivities and specificities of CE MRA and CDUS in the evaluation of the degree of CS were compared, and their advantages, disadvantages and diagnostic value were evaluated. The results obtained may serve as a guide in the clinical selection of examination, diagnostic and treatment methods for CS.

\section{Materials and methods}

General information. A total of 54 patients presenting with CS were admitted to the First Affiliated Hospital of Xinxiang Medical University (Xinxiang, China) from January 2012 to January 2014. All patients presented with dizziness and discomfort, which, from clinical evaluation, were suspected to arise from vascular diseases in the head and neck. In addition, all patients had experienced transient ischemic attack and other neurological symptoms, including high blood pressure, diabetes, hyperlipidemia, cerebral infarction and limb weakness, within the last 6 months. The patients consisted of 32 males and 22 females aged between 37 and 82 years, with a mean age of $63.06 \pm 13.21$ years. All patients underwent CDUS, CE MRA and DSA examinations within 1 week of diagnosis. The current study was conducted in accordance with the Declaration of Helsinki and with approval from the Ethics Committee of the First Affiliated Hospital of Xinxiang Medical University. Written informed consent for participation in the present study was obtained from all patients.

Examination. CE MRA was performed using a SIGNA 3.0T superconducting magnetic resonance imaging (MRI) system (GE Healthcare Bio-sciences, Pittsburgh, PA, USA) equipped with a head-neck joint coil, as described previously (13). Intravenous access was established prior to scanning via the elbow vein using a high pressure syringe. The enhanced MRA used a 3D time-resolved imaging of contrast kinetics (TRICKS) sequence, and the scanning range involved all cervical vessels, including the Willis circle and aortic arch. CE MRA scanning parameters were as follows: Repetition time, $3.6 \mathrm{msec}$; echo time, $0.9 \mathrm{msec}$; flip angle, $30^{\circ}$; layer thickness, $2.8 \mathrm{~mm}$; matrix, $320 \times 180$; and field of view, $40 \times 30 \mathrm{~cm}$. The mask was first scanned for $18 \mathrm{sec}$, then a high-pressure syringe was used for rapid intravenous injection of $30 \mathrm{ml}$ gadolinium diethylenetriamine penta-acetic acid, as an MRI contrast agent, at a rate of $2.5 \mathrm{ml} / \mathrm{sec}$. Saline $(20 \mathrm{ml})$ was then injected at the same rate. At 4-6 sec after injection of the contrast agent, simultaneous 3D TRICKS scanning was initiated, with a scanning time of $1 \mathrm{~min}$ and $24 \mathrm{sec}$, providing 14 phases that each generated 60 images. When scanning was complete, the computer system automatically performed subtractions to reconstruct the vascular images. Using the MRI post-processing workstation, the 'pure' arterial phase was selected to exhibit the optimal phase. Maximum intensity projection and virtual reality image-processing software (Advanced workstation, version 4.4; GE Healthcare Biosciences) was used to generate $15^{\circ}$-interval images along the head-end axis. Blood vessel images were rotated in all directions to achieve optimum lesion display.

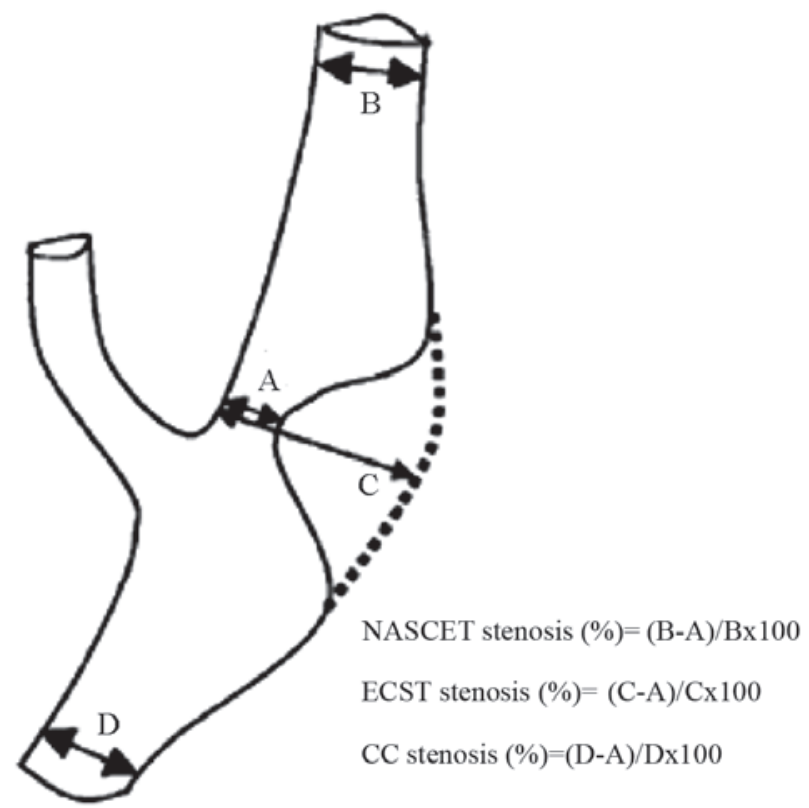

Figure 1. Different assessment methods of ICA stenosis. (A) is the narrowest region of the lumina, $(\mathrm{B})$ is the normal ICA lumina diameter where the distal walls of the bulbar zone are parallel, (C) is the estimated original ICA lumina diameter of the narrowest region and (D) is the normal common carotid artery lumina diameter where the walls near the bulbar zone are parallel. ICA, internal carotid artery; NASCET, North American Symptomatic Carotid Endarterectomy Trial method; ECST, European Carotid Surgery Trial method; CC, common carotid method.

A Color Doppler Ultrasography (CDUS) diagnostic instrument (Esaote North America, Inc., Indianapolis, IN USA) with a probe frequency range of 5-12 $\mathrm{MHz}$ was used for CDUS examinations. The examination was performed on the cervical carotid artery, internal carotid artery, external carotid artery and vertebral artery. Real-time imaging was combined with color Doppler blood flow and spectral information to diagnose vascular lesions, as described previously (14).

CS grading and diagnostic criteria. CS assessment is generally performed using three methods (Fig. 1), namely, the European Carotid Surgery Trial method (15), the North American Symptomatic Carotid Endarterectomy Trial (NASCET) symptomatic CS grading scale (15) and the European Carotid Surgery Trial method (16). U-King-Im et al (17) evaluated the use of enhanced MRA in CS and concluded that all three assessment methods were suitable for DSA techniques. However, their research preferentially supported the use of the NASCET method due to its improved sensitivity in diagnosing severe stenosis (17). Therefore, in the present study, the NASCET scale was used as the diagnostic method for the grading of stenosis as follows: 0, normal (0\%); mild (1-49\%); moderate (50-69\%); severe (70-99\%); and occluded (100\%) (15). A total of four vessels, namely the bilateral common carotid arteries and the bilateral internal carotid arteries, were examined in each of the 54 patients. Thus, a total of 216 vessels were examined. For all vessel characterizations, the CS lesions and degree of vascular stenosis were jointly detected and diagnosed by two senior diagnostic-imaging physicians, who were blinded to the results of other tests. Three experienced radiologists were involved in the ultrasound examinations. 
Table I. Comparison of diagnostic results from CE MRA and DSA.

\begin{tabular}{lrrccc}
\hline \multirow{2}{*}{$\begin{array}{l}\text { Degree of } \\
\text { stenosis by }\end{array}$} & \multicolumn{5}{c}{ Degree of stenosis by DSA } \\
\cline { 2 - 6 } CE MRA & Normal & Mild & Moderate & Severe & Occluded \\
\hline Normal & 141 & 8 & 0 & 0 & 0 \\
Mild & 6 & 45 & 0 & 0 & 0 \\
Moderate & 0 & 2 & 8 & 0 & 0 \\
Severe & 1 & 0 & 0 & 5 & 0 \\
Occluded & 0 & 0 & 0 & 0 & 0 \\
\hline
\end{tabular}

Data represent the vessel numbers $(n=216)$ diagnosed with varying degrees of carotid stenosis by CE MRA and DSA. CE MRA, color-enhanced magnetic resonance angiography; DSA, digital subtraction angiography.

Table II. Comparison of diagnostic results from CDUS and DSA.

\begin{tabular}{lrrccc}
\hline $\begin{array}{l}\text { Degree of } \\
\text { stenosis } \\
\text { by CDUS }\end{array}$ & \multicolumn{5}{c}{ Degree of stenosis by DSA } \\
\cline { 2 - 6 } & Normal & Mild & Moderate & Severe & Occluded \\
\hline Normal & 117 & 12 & 4 & 0 & 0 \\
Mild & 31 & 37 & 2 & 0 & 0 \\
Moderate & 0 & 3 & 1 & 0 & 0 \\
Severe & 0 & 2 & 1 & 5 & 0 \\
Occluded & 0 & 1 & 0 & 0 & 0 \\
\hline
\end{tabular}

Data represents the vessel numbers $(n=216)$ diagnosed with varying degrees of carotid stenosis by CDUS and DSA. CDUS, color Doppler ultrasound; DSA, digital subtraction angiography.

Statistical analysis. Statistical analysis was performed using SPSS 13.0 software (SPSS, Inc., Chicago, IL, USA). The DSA diagnostic results were set as the standard, against which the sensitivities and specificities (sensitivity $=$ true positive rate, and specificity $=$ true negative rate based on DSA diagnosis) of CE MRA and CDUS in diagnosing different degrees of CS were determined. The detection rates of all three imaging methods in detecting carotid artery plaques were compared using a $\chi^{2}$ test and $\mathrm{P}<0.05$ was considered to indicate a statistically significant difference.

\section{Results}

CE MRA diagnosis. The consistency of diagnostic classifications determined by CE MRA and DSA was $92.13 \%$ (199/216 vessels). In 4.17\% vessels (9/216), the degree of stenosis was overestimated by CE MRA, while the degree of stenosis in $3.70 \%$ vessels $(8 / 216)$ was underestimated (Table I).

CDUS diagnosis. The diagnostic accuracy of CDUS, based on the diagnostic results of DSA, was $74.07 \%$ (160/216 vessels).
In $17.59 \%$ vessels (38/216), the degree of stenosis was overestimated by CDUS, while in $8.33 \%$ vessels (18/216), the degree of stenosis was underestimated (Table II).

Sensitivities and specificities of CE MRA and CDUS in the diagnosis of mild stenosis. Among the 55 vessels that were diagnosed with mild stenosis (stenosis degree, 1-49\%) by DSA, the sensitivity and specificity of CE MRA determinations were 85.45 and $95.27 \%$, respectively, while those of CDUS were 78.18 and $79.05 \%$, respectively.

Sensitivities and specificities of CE MRA and CDUS in the diagnosis of moderate stenosis. Among the 8 vessels that were diagnosed with moderate stenosis (stenosis degree, 50-69\%) by DSA, the sensitivity and specificity of CE MRA were 100 and $98.58 \%$, respectively, whereas those of CDUS were 50 and $93.36 \%$, respectively.

Sensitivities and specificities of CE MRA and CDUS in the diagnosis of severe stenosis. Among the 5 vessels that were diagnosed by DSA with severe stenosis (stenosis degree, 70-99\%; Fig. 2), mild stenosis was observed in the left common carotid artery and moderate stenosis in the left internal carotid artery. The left external carotid artery displayed severe stenosis. The sensitivities of CE MRA and CDUS were both $100 \%$ and the specificities of CE MRA and CDUS were 99.53 and $98.10 \%$, respectively.

Detection rates for carotid artery plaque. As depicted in Table III, the detection rate of CDUS for carotid sinus plaques was $48.15 \%$, which was significantly higher than $8.33 \%$ of DSA and $6.48 \%$ of CE MRA, respectively $(\mathrm{P}<0.001)$. The detection rates of CDUS for common carotid artery plaques and internal carotid artery plaques were 39.81 and $31.48 \%$, respectively, which was not significantly different compared with DSA (28.70 and 32.41\%) and CE MRA (26.85 and $30.56 \%)$, respectively $(\mathrm{P}>0.05)$.

\section{Discussion}

The development of imaging technology has enabled improvements in the techniques available for the diagnosis of CS. For instance, carotid ultrasound, MRA and DSA are able to determine the location and degree of CS, as well as the conditions of the surrounding wall-adherent plaques $(18,19)$. Non-invasive imaging techniques, including MRA and CDUS, are now widely available, and are replacing DSA in the diagnosis of CS, though the accuracy of these techniques remains unclear. In a meta-analysis of CS patients presenting with symptoms of carotid territory ischemia conducted by Wardlaw et al (20), the accuracy of non-invasive imaging techniques compared with intra-arterial angiography in the diagnosis of CS was evaluated, and it was concluded that CE MRA has higher sensitivity and specificity than Doppler ultrasound in the diagnosis of severe stenosis. However, data regarding the diagnosis of moderate stenosis and the diagnostic value of combined non-invasive tests are limited and unreliable. A previous study has documented that enhanced MRA may be accurate in identifying carotid artery occlusions, which, once diagnosed, do not require additional examinations (21). Furthermore, 
Table III. Detection of carotid artery plaques by DSA, CE MRA and CDUS.

Plaque detection rate, number of vessels $(\%)$

\begin{tabular}{|c|c|c|c|c|c|}
\hline \multirow[b]{2}{*}{$\begin{array}{l}\text { Examination } \\
\text { method }\end{array}$} & \multirow[b]{2}{*}{$\begin{array}{c}\text { Number of } \\
\text { patients }\end{array}$} & \multirow{2}{*}{$\begin{array}{c}\text { Number of } \\
\text { blood vessels } \\
\text { (2/patient) }\end{array}$} & \\
\hline & & & $\begin{array}{c}\text { Common } \\
\text { carotid artery }\end{array}$ & $\begin{array}{l}\text { Carotid } \\
\text { sinus }\end{array}$ & $\begin{array}{c}\text { Internal carotid } \\
\text { artery }\end{array}$ \\
\hline DSA & 54 & 108 & $31(28.70)$ & $9(8.33)$ & $35(32.41)$ \\
\hline CE MRA & 54 & 108 & $29(26.85)$ & $7(6.48)$ & $33(30.56)$ \\
\hline CDUS & 54 & 108 & $43(39.81)$ & $52(48.15)$ & $34(31.48)$ \\
\hline$\chi^{2}$ & & & 4.896 & 72.178 & 0.086 \\
\hline P-value & & & 0.086 & $<0.001$ & 0.958 \\
\hline
\end{tabular}

DSA, digital subtraction angiography; CE MRA, contrast-enhanced magnetic resonance angiography; CDUS, color Doppler ultrasound.
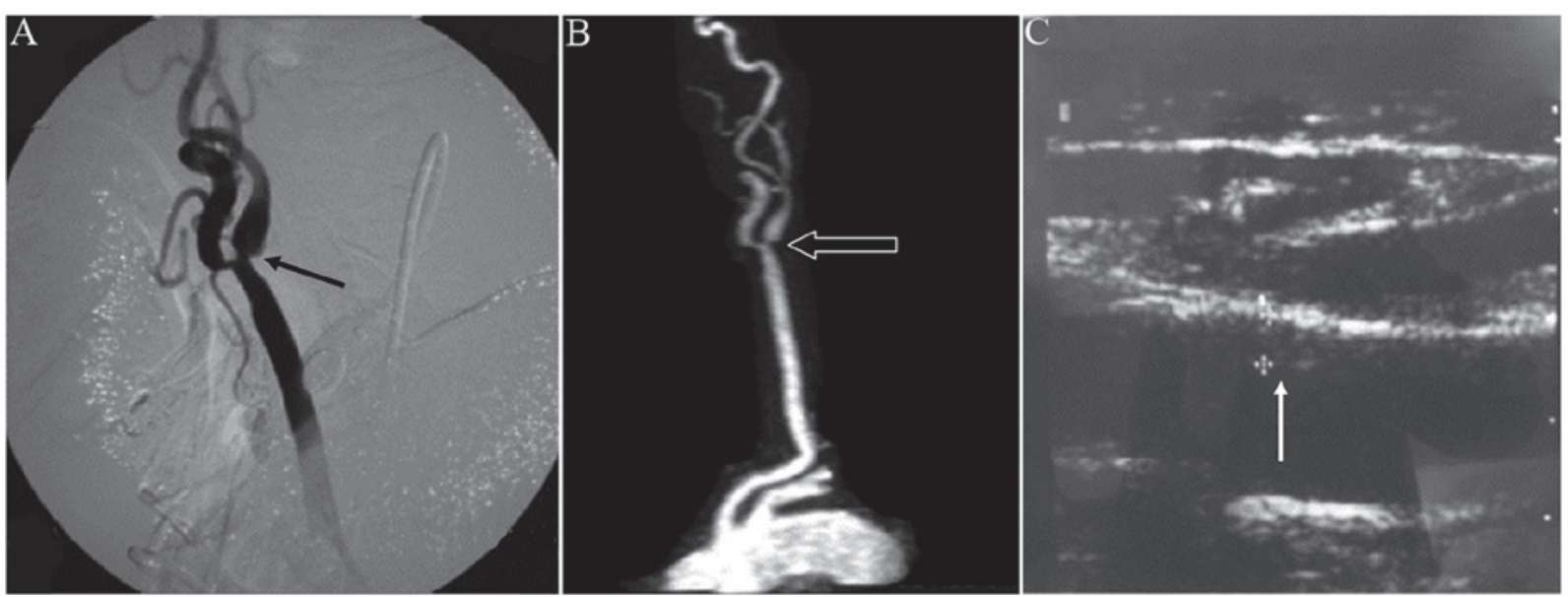

Figure 2. Severe case of CS. (A) Digital subtraction angiography and (B) contrast-enhanced magnetic resonance angiography images from a patient with severe CS exhibiting mild stenosis in the left common carotid artery and moderate stenosis in the left internal carotid artery. Post-stenosis extension is also exhibited. The left external carotid artery displayed severe stenosis. The same degree of stenosis was exhibited in the two examination methods. (C) Color Doppler ultrasound image depicting stenosis of the left common carotid artery as a result of intima thickening. Arrows in A, B and C indicated the moderate stenosis, severe stenosis and intima thickening, respectively. CS, carotid stenosis.

it is suggested that enhanced MRA may replace DSA as a diagnostic tool $(18,22)$. Therefore, the present study aimed to elucidate the optimal noninvasive alternative to DSA, by comparing the diagnostic accuracy and plaque detection rates of CE MRA, CDUS and DSA in 52 patients with CS.

It was observed that the sensitivity of CE MRA was significantly higher than that of CDUS in the detection of mild and moderate degrees of stenosis. This may be due to the lower resolution and greater technical dependence of CDUS. The sensitivities of CE MRA and CDUS in the detection of severe stenosis were both $100 \%$, indicating that the accuracy of these methods in assessing severe CS is similar to that of DSA. The false-negative results obtained by CE MRA may be due to the lower resolution of MRA images, as well as the awkward positioning of the carotid bifurcation. The false-negative results obtained by CDUS may also be related to the lower resolution images, as well as the operator's skill.

The specificity of CE MRA in the detection of mild stenosis was superior to that of CDUS, possibly due to the signal interference caused by plaque and medial thickening in CDUS. High rates of false positive results are generally considered to be a disadvantage, due to unnecessary follow-up examinations, which, in the case of CS, are invasive and dangerous surgical procedures, particularly regarding DSA. In some cases, lower sensitivity may be preferable, as false negatives are later detected in subsequent routine examinations. However, the use of CDUS may be justified by its low cost and simplicity. In addition, the specificities of CE MRA and CDUS in the detection of moderate and severe CS were high.

The varying degrees of false-positive stenosis results generated by CE MRA may be due to the following factors: i) Different sizes of the carotid sinus leading to difficulties distinguishing between normal and narrow widths, ii) poor image quality and/or partial stenosis causing partial signal loss and iii) awkward positioning of lesions.

Previous results have indicated that CDUS evaluations generally overestimate the degree of stenosis (23). In the current study, some false-positive results were generated by CDUS, possibly due to differences in the imaging principles for CDUS and DSA. Images from CDUS reveal the structures of vascular walls and lumens, and provide enhanced visualization of plaques (Fig. 2). This enhanced imaging allows intravascular membrane structure and thickness to be observed, and thus may lead to an increased positive diagnosis 
rate. Importantly, CE MRA can be used to examine the carotid artery from the aortic arch to the Willis circle and so is able to detect non-continuous stenosis. CDUS is the pulse Doppler technique and the $2 \mathrm{MHz}$ emission frequency that makes ultrasound. The sound beam is able to penetrate the thinner part of the skull and directly map the blood flow to the lower part of the brain. The Doppler signal, which takes the hemodynamic parameters of the base of the cerebellum, reflects brain blood vascular stenosis. DSA is a novel X-ray imaging system that is conventional angiography and electricity. It is the combination of computer image processing technology. The diagnosis of cerebral blood vessels can clearly show the presence of brain angiography, not just normal. Blood vessels, diseased vessels and narrow, obstructive blood vessels are also diagnosed. In addition, the operator's skill does not affect diagnosis by enhanced MRA, while results obtained by CDUS are strongly dependent on the observer (24). A previous comparative study of DSA evaluated the diagnostic accuracy of CDUS, CT angiography (CTA) and blood-pool-enhanced MRA in the assessment of 170 patients with CS. Consistent with the present study findings, it was observed that CTA was the most accurate technique for evaluating CS, with CTA exhibiting a marginally higher accuracy than MRA ( $97 \%$ for CTA vs. 95\% for steady-state MRA and $92 \%$ for first-pass MRA) and a markedly greater accuracy than CDUS (97 vs. $76 \%$, respectively) (25).

In addition to the established contraindications of MRA such as cardiac pacemaker, cochlear implants, magnetic resonance imaging, brain pacemakers and false teeth, MRI motion artifacts arising from patient movements may limit the accuracy of enhanced MRA. However, due to the short acquisition time, no substantial motion artifact was observed in the present study. Only one case of contrast-agent extravasation due to poor puncturing was observed, and the high-pressure injection was promptly interrupted. The insufficient amount of contrast agent led to poor image quality and thus affected the diagnostic accuracy to a degree.

For one case in the current study, the results of DSA indicated that the left subclavian artery extended from the ascending aorta, which was determined to be an abnormal blood vessel. Thus, the time taken to locate the left subclavian artery for intubation was extended. If the patient had initially undergone MRA, which allows for the rotation of blood vessels in any direction, the direction of blood vessels could have been determined prior to DSA, which would have reduced the damage caused by blind intubation, shortened the inspection time and reduced radiation damage. Therefore, for non-urgent cases, performing MRA prior to DSA may provide additional information to facilitate the performance of DSA.

In conclusion, among the noninvasive CS examinations, CE MRA demonstrated higher sensitivity and specificity than CDUS in the diagnosis of moderate and severe stenosis, while CDUS only exhibited high sensitivity and specificity in the diagnosis of severe stenosis. However, the carotid sinus plaque detection rate of CDUS was significantly higher than that of DSA and CE MRA. Therefore, CDUS may be useful for routine screening, or could be used in combination with CE MRA to determine the nature of carotid plaques. Thus, a combination of MRA and CDUS may be a novel 'gold standard' strategy for the diagnosis of moderate and severe CS. In addition, CE
MRA examination prior to DSA may prevent and/or minimize damage caused by blind intubation during the DSA procedure.

\section{Acknowledgements}

The present study was supported by the National Health and Family Planning Commission of Henan, China (grant no. 201301006).

\section{References}

1. Dix JE, Evans AJ, Kallmes DF, Sobel AH and Phillips CD: Accuracy and precision of CT angiography in a model of carotid artery bifurcation stenosis. AJNR Am J Neuroradiol 18: 409-415, 1997.

2. Sitzer M, Müller W, Siebler M, Hort W, Kniemeyer HW, Jäncke L and Steinmetz H: Plaque ulceration and lumen thrombus are the main sources of cerebral microemboli in high-grade internal carotid artery stenosis. Stroke 26: 1231-1233, 1995.

3. Flaherty ML, Kissela B, Khoury JC, Alwell K, Moomaw CJ, Woo D, Khatri P, Ferioli S, Adeoye O, Broderick JP and Kleindorfer D: Carotid artery stenosis as a cause of stroke. Neuroepidemiology 40: 36-41, 2013.

4. Ricotta JJ, Aburahma A, Ascher E, Eskandari M, Faries P, Lal BK and Society for Vascular Surgery: Updated society for vascular surgery guidelines for management of extracranial carotid disease. J Vasc Surg 54: e1-e31, 2011.

5. Debrey SM, Yu H, Lynch JK, Lövblad KO, Wright VL, Janket SJ and Baird AE: Diagnostic accuracy of magnetic resonance angiography for internal carotid artery disease: A systematic review and meta-analysis. Stroke 39: 2237-2248, 2008.

6. Zhao H, Wang J, Liu X, Zhao X, Hippe DS, Cao Y, Wan J, Yuan C and $\mathrm{Xu}$ J: Assessment of carotid artery atherosclerotic disease by using three-dimensional fast black-blood MR imaging: Comparison with DSA. Radiology 274: 508-516, 2015.

7. Elias RM, Wald JT and Kallmes DF: Diagnosis of carotid artery stenosis with oculopneumoplethysmography alone and in combination with MRA. Vasc Health Risk Manag 8: 631-639, 2012.

8. Guédon A, Clarençon F, Di Maria F, Rosso C, Biondi A, Gabrieli J, Rojas P, Chiras J and Sourour N: Very late ischemic complications in flow-diverter stents: A retrospective analysis of a single-center series. J Neurosurg 125: 929-935, 2016.

9. D'Onofrio M, Mansueto G, Faccioli N, Guarise A, Tamellini P, Bogina G and Pozzi Mucelli R: Doppler ultrasound and contrast-enhanced magnetic resonance angiography in assessing carotid artery stenosis. Radiol Med 111: 93-103, 2006 (In English, Italian).

10. Anzidei M, Napoli A, Marincola BC, Nofroni I, Geiger D, ZaccagnaF,Catalano Cand Passariello R: Gadofosveset-enhanced MR angiography of carotid arteries: Does steady-state imaging improve accuracy of first-pass imaging? Comparison with selective digital subtraction angiography. Radiology 251: 457-466, 2009.

11. Goyal M, Nicol J and Gandhi D: Evaluation of carotid artery stenosis: Contrast enhanced magnetic resonance angiography compared with conventional digital subtraction angiography. Can Assoc Radiol J 55: 111-119, 2004.

12. Qureshi AI, Suri MF, Ali Z, Kim SH, Fessler RD, Ringer AJ, Guterman LR, Budny JL and Hopkins LN: Role of conventional angiography in evaluation of patients with carotid artery stenosis demonstrated by Doppler ultrasound in general practice. Stroke 32: 2287-2291, 2001.

13. Petkova M, Gauvrit JY, Trystram D, Nataf F, Godon-Hardy S, Munier T, Oppenheim C and Meder JF: Three-dimensional dynamic time-resolved contrast-enhanced MRA using parallel imaging and a variable rate k-space sampling strategy in intracranial arteriovenous malformations. J Magn Reson Imaging 29: 7-12, 2009.

14. Accorsi F: Color Doppler of the extracranial and intracranial arteries in the acute phase of cerebral ischemia. J Ultrasound 16: 187-193, 2013.

15. Adam G, Kocak E, Özkan A, Reşorlu M, Çınar C, Bozkaya H, Kurt T, Altun B, Şen HM, Akbal A, et al: Ȩvaluation of platelet distribution width and mean platelet volume in patients with carotid artery stenosis. Angiology 66: 375-378, 2015. 
16. Rothwell PM, Gutnikov SA, Warlow CP and European Carotid Surgery Trialist's Collaboration: Reanalysis of the final results of the european carotid surgery trial. Stroke 34: 514-523, 2003.

17. U-King-ImJM, Trivedi RA, Cross JJ, Higgins NJ,Hollingworth W, Graves M, Joubert I, Kirkpatrick PJ, Antoun NM and Gillard JH: Measuring carotid stenosis on contrast-enhanced magnetic resonance angiography: Diagnostic performance and reproducibility of 3 different methods. Stroke 35: 2083-2088, 2004.

18. Kubale R and Arning C: Significance of Doppler ultrasound procedures for diagnosis of carotid stenosis. Radiologe 44: 946-949, 2004 (In German).

19. Honish C, Sadanand V, Fladeland D, Chow V and Pirouzmand F: The reliability of ultrasound measurements of carotid stenosis compared to MRA and DSA. Can J Neurol Sci 32: 465-471, 2005.

20. Wardlaw JM, Chappell FM, Best JJ, Wartolowska K and Berry E; NHS Research and Development Health Technology Assessment Carotid Stenosis Imaging Group: Non-invasive imaging compared with intra-arterial angiography in the diagnosis of symptomatic carotid stenosis: A meta-analysis. Lancet 367 : 1503-1512, 2006.

21. Hammond CJ, McPherson SJ, Patel JV and Gough MJ: Assessment of apparent internal carotid occlusion on ultrasound Prospective comparison of contrast-enhanced ultrasound, magnetic resonance angiography and digital subtraction angiography. Eur J Vasc Endovasc Surg 35: 405-412, 2008.
22. Naganawa S, Koshikawa T, Fukatsu H, Sakurai Y, Ichinose N, Ishiguchi T and Ishigaki T: Contrast-enhanced MR angiography of the carotid artery using 3D time-resolved imaging of contrast kinetics: Comparison with real-time fluoroscopic triggered 3D-elliptical centric view ordering. Radiat Med 19: 185-192, 2001.

23. Netuka D, Ostrý S, Belsán T, Rucka D, Mandys V, Charvát F, Bradác $\mathrm{O}$ and Benes $\mathrm{V}$ : Magnetic resonance angiography, digital subtraction angiography and Doppler ultrasonography in detection of carotid artery stenosis: A comparison with findings from histological specimens. Acta Neurochir (Wien) 152: 1215-1221, 2010.

24. Mead GE, Lewis SC and Wardlaw JM: Variability in Doppler ultrasound influences referral of patients for carotid surgery. Eur J Ultrasound 12: 137-143, 2000.

25. Anzidei M, Napoli A, Zaccagna F, Di Paolo P, Saba L, Cavallo Marincola B, Zini C, Cartocci G, Di Mare L, Catalano C and Passariello R: Diagnostic accuracy of colour Doppler ultrasonography, CT angiography and blood-pool-enhanced MR angiography in assessing carotid stenosis: A comparative study with DSA in 170 patients. Radiol Med 117: 54-71, 2012. 